\title{
On a redundancy in the parity-violating 2-nucleon contact Lagrangian
}

\author{
L. Girlanda* \\ INFN, Sez. di Pisa, Largo Bruno Pontecorvo, 56127 Pisa, Italy
}

(Dated: August 18, 2021)

\begin{abstract}
It is shown that the number of independent operators in the nucleon-nucleon parity violating contact Lagrangian at the leading order in the low-momentum expansion, can be reduced to five, by using Fierz rearrangements of the nucleon fields.
\end{abstract}

PACS numbers: 11.30.Er 12.39.Fe,21.30.Fe,21.45.-v

*girlanda@pi.infn.it 
Recent interest in the study of hadronic parity violation has been triggered by the planning of new experiments involving few-body systems, like NPDGamma at LANSCE [1] or neutron spin rotation on ${ }^{4} \mathrm{He}$ at NIST [2], in conjunction with the enormous progress in the theoretical control on ab-initio calculations on such systems [3]. The traditional theoretical framework for such studies was set by Desplanques, Donoghue and Holstein in Ref. [4], in the form of a meson exchange model, generally known as the DDH model. More recently an effective field theory description of nuclear parity violation has been proposed in Ref. [5, 6], in which nucleons and pions interact according to the dictates of chiral symmetry (see Ref. [7] for a comprehensive review). At sufficiently low energies, pions can be integrated out and their contribution (as well as the ones from higher-mass particles) is subsumed in the values of the coupling constants of the contact interactions among nucleons ("pionless effective field theory"). In either case the Lagrangian containing the nucleon contact interactions appears as a crucial ingredient in the effective field theory.

In the heavy baryon formalism the most general Lagrangian yields redundant operators, which have to be eliminated by imposing reparameterization invariance [8]. Alternatively, one can start from the relativistic theory and perform the non-relativistic reduction afterwards, as was done in Ref. [5].

The two-nucleon contact interactions can be classified according to their transformation properties under the chiral symmetry [9]. The possible flavor structures that can arise change the isospin by $\Delta I=0,1,2$ :

$$
\begin{array}{ll}
F_{1}^{i j, k l}=\delta_{i j} \delta_{k l}, \quad \Delta I=1: \quad & F_{2^{\prime}}^{i j, k l}=\delta_{i j} \tau_{k l}^{3}, \\
F_{4^{\prime}}^{i j, k l}=\tau_{i j}^{3} \delta_{k l}, \quad \Delta I=2: \quad F_{5}^{i j, k l}=\mathcal{I}^{a b} \tau_{i j}^{a} \tau_{k l}^{b}, \\
F_{6}^{i j, k l}=i \epsilon^{a b 3} \tau_{i j}^{a} \tau_{k l}^{b},
\end{array}
$$

where $i, j, k, l$ are the isospin indices of the four nucleons and the matrix $\mathcal{I}=\operatorname{diag}(1,1,-2)$. Notice that all these structures are symmetric under the exchange of $i \leftrightarrow j$ and $k \leftrightarrow l$, except $F_{6}^{i j, k l}$, which is antisymmetric. It is also convenient to classify the flavor structures according to the symmetry properties under the exchange of $(i j) \leftrightarrow(k l)$ and use, instead of $F_{2^{\prime}}^{i j, k l}$ and $F_{4^{\prime}}^{i j, k l}$, the structures

$$
F_{2}^{i j, k l}=\delta_{i j} \tau_{k l}^{3}+\delta_{k l} \tau_{i j}^{3}, \quad F_{4}^{i j, k l}=\delta_{i j} \tau_{k l}^{3}-\delta_{k l} \tau_{i j}^{3}
$$


so that

$$
F_{i}^{i j, k l}=s_{i} F_{i}^{k l, i j}, \quad s_{1}=s_{2}=s_{3}=-s_{4}=s_{5}=-s_{6}=1 .
$$

One can easily list all possible Lorentz invariant four-nucleon operators which violate $\mathrm{P}$ and conserve CP. Those containing up to one space-time derivative are listed below $(i=$ $1, \ldots, 5$ henceforth),

$$
\begin{array}{ll}
O_{i}^{(1)}=F_{i} \otimes \bar{\psi} \gamma^{\mu} \psi \bar{\psi} \gamma_{\mu} \gamma_{5} \psi, & O_{6}^{(2)}=F_{6} \otimes \bar{\psi} \psi \bar{\psi} \gamma_{5} \psi, \\
\tilde{O}_{i}^{(1)}=F_{i} \otimes \bar{\psi} i \overleftrightarrow{\partial}_{\mu} \psi \bar{\psi} \gamma^{\mu} \gamma_{5} \psi, & \tilde{O}_{6}^{(5)}=F_{6} \otimes \bar{\psi} \gamma_{5} i \overleftrightarrow{\partial}_{\mu} \psi \bar{\psi} \gamma^{\mu} \psi, \\
\tilde{O}_{i}^{(2)}=F_{i} \otimes \bar{\psi} \gamma^{\mu} \gamma_{5} \psi \partial^{\nu}\left(\bar{\psi} \sigma_{\mu \nu} \psi\right), & \tilde{O}_{6}^{(6)}=F_{6} \otimes \bar{\psi} \gamma^{\mu} \psi \partial^{\nu}\left(\bar{\psi} \sigma_{\mu \nu} \gamma_{5} \psi\right), \\
\tilde{O}_{i}^{(3)}=F_{i} \otimes \bar{\psi} \gamma_{\mu} \overleftrightarrow{\partial}_{\nu} \psi \bar{\psi} \sigma^{\mu \nu} \gamma_{5} \psi, & \tilde{O}_{6}^{(7)}=F_{6} \otimes \bar{\psi} \gamma_{\mu} \gamma_{5} \stackrel{\leftrightarrow}{\partial}{ }_{\nu} \psi \bar{\psi} \sigma^{\mu \nu} \psi, \\
\tilde{O}_{i}^{(4)}=F_{i} \otimes \bar{\psi} \gamma_{\mu} \psi \bar{\psi} \overleftrightarrow{\partial}_{\nu} \sigma^{\mu \nu} \gamma_{5} \psi, & \tilde{O}_{6}^{(8)}=F_{6} \otimes \bar{\psi} \gamma_{\mu} \gamma_{5} \psi \bar{\psi} \overleftrightarrow{\partial}_{\nu} \sigma^{\mu \nu} \psi
\end{array}
$$

Some of these operators are related by the field equations of motion. Using the field equations of motion to reduce the number of independent operators corresponds to the freedom of redefining the interpolating fields of the effective theory. One can establish the following relations,

$$
\begin{aligned}
& \tilde{O}_{i}^{(2)}=s_{i}\left[2 m O_{i}^{(1)}-\tilde{O}_{i}^{(1)}\right] \\
& \tilde{O}_{i}^{(3)}=-\tilde{O}_{i}^{(2)} \\
& \tilde{O}_{i}^{(4)}=-2 m O_{i}^{(1)} \\
& \tilde{O}_{6}^{(5)}=\tilde{O}_{6}^{(6)} \\
& \tilde{O}_{6}^{(7)}=-\tilde{O}_{6}^{(6)} \\
& \tilde{O}_{6}^{(8)}=2 m O_{6}^{(2)}
\end{aligned}
$$

so that everything can be expressed as combinations of the 12 operators $O_{1, \ldots, 5}^{(1)}, O_{6}^{(2)}, \tilde{O}_{1, \ldots, 5}^{(2)}$ and $\tilde{O}_{6}^{(6)}$, which correspond to the set identified in Appendix A of Ref. [5]. Based on the above set of operators, a non-relativistic Lagrangian containing 11 operators was introduced (cfr. Eq. (71) of Ref. [5]), and used to derive a potential containing 10 operators (cfr. Eq. (5) of Ref. [5] or Eq. (9) of Ref. [6]).

This set of operators is complete but redundant, as can be shown by using Fierz rearrangements. Let us start with the flavor structures. By interchanging the indices $j$ and $l$, the following relations hold:

$$
2 F_{1}^{i l, k j}=F_{1}^{i j, k l}+F_{3}^{i j, k l}
$$




$$
\begin{aligned}
& F_{2}^{i l, k j}=F_{2}^{i j, k l}, \\
& F_{4}^{i l, k j}=F_{6}^{i j, k l}, \\
& F_{5}^{i l, k j}=F_{5}^{i j, k l} .
\end{aligned}
$$

For the spinor structures of interest, the relevant Fierz identities [10] are conveniently written as

$$
\begin{aligned}
()\left[\gamma_{5}\right]= & \frac{1}{4}\left\{\left(\gamma_{5}\right][)+(]\left[\gamma_{5}\right)+\left(\gamma^{\mu} \gamma_{5}\right]\left[\gamma_{\mu}\right)-\left(\gamma^{\mu}\right]\left[\gamma_{\mu} \gamma_{5}\right)+\frac{1}{2}\left(\sigma^{\mu \nu}\right]\left[\sigma_{\mu \nu} \gamma_{5}\right)\right\} \\
\left(\gamma^{\mu}\right)\left[\gamma_{\mu} \gamma_{5}\right]= & \left(\gamma_{5}\right][)-(]\left[\gamma_{5}\right)-\frac{1}{2}\left(\gamma^{\mu} \gamma_{5}\right]\left[\gamma_{\mu}\right)-\frac{1}{2}\left(\gamma^{\mu}\right]\left[\gamma_{\mu} \gamma_{5}\right) \\
\left(\sigma_{\mu \nu}\right)\left[\gamma^{\mu} \gamma_{5}\right]= & \frac{3}{4} i\left\{-\left(\gamma_{\nu} \gamma_{5}\right][)+\left(\gamma_{\nu}\right]\left[\gamma_{5}\right)+\left(\gamma_{5}\right]\left[\gamma_{\nu}\right)+(]\left[\gamma_{\nu} \gamma_{5}\right)\right\} \\
& +\frac{1}{4}\left\{-\left(\sigma_{\mu \nu}\right]\left[\gamma^{\mu} \gamma_{5}\right)-\left(\gamma^{\mu} \gamma_{5}\right]\left[\sigma_{\mu \nu}\right)-\left(\gamma_{5} \sigma_{\mu \nu}\right]\left[\gamma^{\mu}\right)+\left(\gamma^{\mu}\right]\left[\gamma_{5} \sigma_{\mu \nu}\right)\right\}, \\
()\left[\gamma_{\nu} \gamma_{5}\right]= & \frac{1}{4}\left\{\left(\gamma_{\nu} \gamma_{5}\right][)-\left(\gamma_{\nu}\right]\left[\gamma_{5}\right)+\left(\gamma_{5}\right]\left[\gamma_{\nu}\right)+(]\left[\gamma_{\nu} \gamma_{5}\right)\right\} \\
& +\frac{i}{4}\left\{-\left(\sigma_{\mu \nu}\right]\left[\gamma^{\mu} \gamma_{5}\right)+\left(\gamma^{\mu} \gamma_{5}\right]\left[\sigma_{\mu \nu}\right)-\left(\gamma_{5} \sigma_{\mu \nu}\right]\left[\gamma^{\mu}\right)-\left(\gamma^{\mu}\right]\left[\gamma_{5} \sigma_{\mu \nu}\right)\right\}, \\
\left(\gamma^{\mu}\right)\left[\gamma_{5} \sigma_{\mu \nu}\right]= & \frac{3}{4} i\left\{\left(\gamma_{\nu} \gamma_{5}\right][)+\left(\gamma_{\nu}\right]\left[\gamma_{5}\right)-\left(\gamma_{5}\right]\left[\gamma_{\nu}\right)+(]\left[\gamma_{\nu} \gamma_{5}\right)\right\} \\
& +\frac{1}{4}\left\{\left(\sigma_{\mu \nu}\right]\left[\gamma^{\mu} \gamma_{5}\right)-\left(\gamma^{\mu} \gamma_{5}\right]\left[\sigma_{\mu \nu}\right)-\left(\gamma_{5} \sigma_{\mu \nu}\right]\left[\gamma^{\mu}\right)-\left(\gamma^{\mu}\right]\left[\gamma_{5} \sigma_{\mu \nu}\right)\right\}, \\
& \frac{1}{4}\left\{-\left(\gamma_{\nu} \gamma_{5}\right][)+\left(\gamma_{\nu}\right]\left[\gamma_{5}\right)+\left(\gamma_{5}\right]\left[\gamma_{\nu}\right)+(]\left[\gamma_{\nu} \gamma_{5}\right)\right\} \\
& +\frac{i}{4}\left\{-\left(\sigma_{\mu \nu}\right]\left[\gamma^{\mu} \gamma_{5}\right)-\left(\gamma^{\mu} \gamma_{5}\right]\left[\sigma_{\mu \nu}\right)-\left(\gamma_{5} \sigma_{\mu \nu}\right]\left[\gamma^{\mu}\right)+\left(\gamma^{\mu}\right]\left[\gamma_{5} \sigma_{\mu \nu}\right)\right\},
\end{aligned}
$$

where "(", ")", "[" and "]" are shorthands for $\bar{\psi}_{1}, \psi_{2}, \bar{\psi}_{3}$ and $\psi_{4}$ respectively and an overall minus sign should be included due do the anticommuting nature of the fermion fields. Combining the flavor and spinor indices rearrangements, we have the operator identities

$$
\begin{aligned}
O_{1}^{(1)} & =O_{3}^{(1)}, \\
O_{4^{\prime}}^{(1)} & =2 O_{6}^{(2)}, \\
4 m O_{1}^{(1)} & =3 \tilde{O}_{1}^{(2)}+\tilde{O}_{3}^{(2)}, \\
\tilde{O}_{2}^{(2)} & =m O_{2}^{(1)} \\
\tilde{O}_{5}^{(2)} & =m O_{5}^{(1)}, \\
\tilde{O}_{4}^{(2)} & =-2 m O_{6}^{(2)}-\tilde{O}_{6}^{(6)} .
\end{aligned}
$$

Thus the number of independent operators is reduced to 6 . 
In the leading order of the non-relativistic reduction, the following relations hold

$$
\begin{aligned}
& O_{i}^{(1)}=\frac{1}{2 m}\left[-N_{i}^{(1)}+s_{i} N_{i}^{(2)}-s_{i} N_{i}^{(3)}\right] \\
& O_{6}^{(2)}=\frac{1}{2 m} N_{6}^{(1)} \\
& \tilde{O}_{i}^{(1)}=-N_{i}^{(1)}+s_{i} N_{i}^{(2)} \\
& \tilde{O}_{i}^{(2)}=-N_{i}^{(3)} \\
& \tilde{O}_{i}^{(3)}=s_{i} N_{i}^{(3)} \\
& \tilde{O}_{i}^{(4)}=N_{i}^{(1)}-s_{i} N_{i}^{(2)}+s_{i} N_{i}^{(3)} \\
& \tilde{O}_{6}^{(5)}=-N_{6}^{(1)} \\
& \tilde{O}_{6}^{(6)}=-N_{6}^{(1)} \\
& \tilde{O}_{6}^{(7)}=N_{6}^{(1)} \\
& \tilde{O}_{6}^{(8)}=N_{6}^{(1)}
\end{aligned}
$$

with the non-relativistic operators defined by

$$
\begin{aligned}
& N_{i}^{(1)}=F_{i} \otimes N^{\dagger} N N^{\dagger} i \stackrel{\leftrightarrow}{\nabla} \cdot \vec{\sigma} N, \\
& N_{i}^{(2)}=F_{i} \otimes N^{\dagger} \vec{\sigma} N \cdot N^{\dagger} i \stackrel{\leftrightarrow}{\nabla} N, \\
& N_{i}^{(3)}=F_{i} \otimes \epsilon_{i j k} N^{\dagger} \sigma^{i} N \nabla^{j}\left(N^{\dagger} \sigma^{k} N\right), \\
& N_{6}^{(1)}=F_{6} \otimes i \vec{\nabla}\left(N^{\dagger} N\right) N^{\dagger} \vec{\sigma} N .
\end{aligned}
$$

One can then make use of the fact that the operators $O_{6}^{(2)}$ and $\tilde{O}_{6}^{(6)}$ give rise to the same nonrelativistic structure, as already observed in Ref. [5]. Therefore, the number of independent operators can be further reduced to 5 up to order $O(Q)$, and the minimal PV two-nucleon non-relativistic contact Lagrangian may be taken to assume the form

$$
\begin{aligned}
\mathcal{L}_{P V, N N}= & \frac{1}{\Lambda_{\chi}^{3}}\left\{C_{1}\left(N^{\dagger} \vec{\sigma} N \cdot N^{\dagger} i \stackrel{\leftrightarrow}{\nabla} N-N^{\dagger} N N^{\dagger} i \stackrel{\leftrightarrow}{\nabla} \cdot \vec{\sigma} N\right)\right. \\
& -\tilde{C}_{1} \epsilon_{i j k} N^{\dagger} \sigma^{i} N \nabla^{j}\left(N^{\dagger} \sigma^{k} N\right) \\
& -C_{2} \epsilon_{i j k}\left[N^{\dagger} \tau_{3} \sigma^{i} N \nabla^{j}\left(N^{\dagger} \sigma^{k} N\right)+N^{\dagger} \sigma^{i} N \nabla^{j}\left(N^{\dagger} \tau_{3} \sigma^{k} N\right)\right] \\
& -\tilde{C}_{5} \mathcal{I}_{a b} \epsilon_{i j k} N^{\dagger} \tau^{a} \sigma^{i} N \nabla^{j}\left(N^{\dagger} \tau^{b} \sigma^{k} N\right) \\
& \left.+C_{6} \epsilon^{a b 3} \vec{\nabla}\left(N^{\dagger} \tau^{a} N\right) \cdot N^{\dagger} \tau^{b} \vec{\sigma} N\right\}
\end{aligned}
$$

where the notations for the coupling constants have been chosen so as to conform to Ref. [5] except for $C_{2}$ which replaces $C_{2}+C_{4}$. Thus, for instance, in the notations of Ref. [5], the 
term

$$
N^{\dagger} \tau^{a} N N^{\dagger} \tau^{a} \vec{\sigma} \cdot i \vec{D}_{-} N-N^{\dagger} \tau^{a} i \vec{D}_{-} N \cdot N^{\dagger} \tau^{a} \vec{\sigma} N, \quad\left[N^{\dagger} i D_{ \pm}^{\mu} N \equiv\left(i D^{\mu} N\right)^{\dagger} N \mp N^{\dagger}\left(i D^{\mu} N\right)\right]
$$

in the non-relativistic reduction of the Lagrangian can be omitted, in as much the same way as a term

$$
N^{\dagger} \tau^{a} \vec{\sigma} N \cdot N^{\dagger} \tau^{a} \vec{\sigma} N
$$

can be omitted in the parity-conserving sector [11].

It is worth noting that the reduction of the number of independent operators down to five has no practical consequences at the present stage of phenomenological analyses since only five combinations of low-energy constants are relevant at low energies. This was already noticed in Refs. [5, 6] on the basis of the observation that, since only S- and Pwave amplitudes are important in this regime, several operators give rise to identical matrix elements (see also the discussion in Ref. [12]). Nevertheless, in view of a description of nuclear parity violation with the chiral effective theory, it is important to use a truly minimal set of operators, and have a one-to-one correspondence between physical observables and low-energy constants of the effective Lagrangian.

I thank Barry Holstein for correspondence, and Rocco Schiavilla and Michele Viviani for useful discussions.

[1] B. Lauss et al., AIP Conf. Proc. 842, 790 (2006) arXiv:nucl-ex/0601004.

[2] C. D. Bass et al., J. Res. Natl. Inst. Stand. Technol. 110, 205 (2005).

[3] M. Viviani, L. E. Marcucci, S. Rosati, A. Kievsky and L. Girlanda, Few Body Syst. 39, 159 (2006) arXiv:nucl-th/0512077.

A. Kievsky, S. Rosati, M. Viviani, L. E. Marcucci, and L. Girlanda, J. Phys. G 35, 063101 (2008) arXiv:0805.4688 [nucl-th]].

V. D. Efros, W. Leidemann, G. Orlandini and N. Barnea, J. Phys. G 34, R459 (2007) arXiv:0708.2803 [nucl-th]].

A. Deltuva and A. C. Fonseca, Phys. Rev. C 76, 021001 (2007) arXiv:nucl-th/0703066; Phys. Rev. Lett. 98, 162502 (2007) arXiv:nucl-th/0703065.

P. Navratil and W. E. Ormand, Phys. Rev. C 68, 034305 (2003) arXiv:nucl-th/0305090. 
W. Gloeckle, H. Witala, D. Huber, H. Kamada and J. Golak, Phys. Rept. 274, 107 (1996).

R. Skibinski, J. Golak, H. Witala, W. Gloeckle, A. Nogga and E. Epelbaum, Acta Phys. Polon.

B 37, 2905 (2006) arXiv:nucl-th/0606021.

J. Carlson and R. Schiavilla, Rev. Mod. Phys. 70, 743 (1998).

K. M. Nollett, S. C. Pieper, R. B. Wiringa, J. Carlson and G. M. Hale, Phys. Rev. Lett. 99, 022502 (2007) arXiv:nucl-th/0612035.

[4] B. Desplanques, J. F. Donoghue and B. R. Holstein, Annals Phys. 124, 449 (1980).

[5] S. L. Zhu, C. M. Maekawa, B. R. Holstein, M. J. Ramsey-Musolf and U. van Kolck, Nucl. Phys. A 748, 435 (2005) arXiv:nucl-th/0407087.

[6] M. J. Ramsey-Musolf and S. A. Page, Ann. Rev. Nucl. Part. Sci. 56, 1 (2006) arXiv:hep-ph/0601127.

[7] E. Epelbaum, Prog. Part. Nucl. Phys. 57, 654 (2006) arXiv:nucl-th/0509032.

[8] M. E. Luke and A. V. Manohar, Phys. Lett. B 286, 348 (1992) arXiv:hep-ph/9205228.

[9] D. B. Kaplan and M. J. Savage, Nucl. Phys. A 556 (1993) 653 [Erratum-ibid. A 570 (1994 ERRAT,A580,679.1994) 833].

[10] J. F. Nieves and P. B. Pal, Am. J. Phys. 72, 1100 (2004) arXiv:hep-ph/0306087.

C. C. Nishi, Am. J. Phys. 73, 1160 (2005) arXiv:hep-ph/0412245].

[11] U. van Kolck, Phys. Rev. C 49, 2932 (1994).

[12] C. P. Liu, Phys. Rev. C 75, 065501 (2007) arXiv:nucl-th/0609078. 